\title{
DEUTSCHE
}

\section{MEDICINISCHE WOCHENSCHRIFT.}

\section{Mit Berïcksichtigung des deutschen Medicinalwesens nach amtlichen Mittheilungen, der öffent- lichen Gesundheitspflege und der Interessen des ärztlichen Standes.}

\author{
Begriindet von Dr. Paul Börner.
}

\section{Einundzwanzigster Jahrgang.}

\author{
Verantwortlicher Redacteur: Prof. Dr. A. Eulenburg, Berlin. - Verlag: Georg Thieme, Leipzig-Berlin. \\ Lichtensteinallee 3 . \\ Postadresse: Leipzig, Seeburgstr. 31.
}

\section{N H A L T.}

Originalartikel: I. Ueber ein neues Heilverfahren bei Netzlautablösung. Von Prof. Dr. R. Deutschmann in Hamburg.

II. Stoffwechselversuch bei Schilddrissenfutterung. Von Dr. L. Bleibtreu und Dr. H. Wendelstadt.

III. Beitrag zur Kenntniss des Papilloma neuropathicum. Von Dr. H. Albers-Schonberg in Leipzig

IV. Retrorectale Dermoidcysten und ibre Exstirpation. Von Dr. Schulze in Wittenberge.

V. Ueber den Versuch einer blutlosen Oberkieferresection durch temporäre Constriction der isolirten Carotis. Von Dr. E. Senger in Crefeld.

VI. Beitrag zur Casuistik der Fremdkörper in der Harnblase. Von Dr. Hochmann in Marienburg.

\section{Ueber ein neues Heilverfahren bei Netz- hautablösung.}

Von Prof. R. Deutschmann in Hamburg.

In einer soeben erschienenen grösseren 'Abhandlung in meinen Beiträgen zur Augenheilkunde ${ }^{1}$ ) habe ich mich bemüht, meinen speziellen Fachgenossen die Berechtigung meines neuen Verfahrens zur Heilung von Netzhautablösung darzulegen, eines Verfahrens, das ich in einer Reihe von Krankheitsfällen dieser Art zu prüfen und schätzen zu lernen Gelegenheit hatte. Bei dem allgemeinen Interesse, das diese schwere Augenerkrankung zweifellos bei allen Aerzten findet, halte ich es für angebracht, in grossen Zügen wenigstens das wichtigste hiervon allgemeinen ärztlichen Kreisen zur Kenntniss zu bringen.

Seit den: gewissenliaften, unter Leitung Leber's gemachten Untersuchungen von Erik Nordenson über die Pathogenese der Netzhautablösung müssen wir annehmen, dass die Netzhautablösung durchweg, von einigen selteneren Formen derselben abgesehen, nicht durch einen primären Erguss von der Aderhaut aus, sondern durch einen Zug von Seiten des schrumpfenden Glaskörpers hervorgebracht wird. Der Glaskörper erhält bei der von Nor d enson genauer beschriebenen chronischen Verdichtung ohne Verlust seiner Durchsichtigkeit eine ausgesprochene lockig fibrilläre Beschaffenheit und zieht sich auf ein immer kleineres Volumen zusammen, wobei der frei werdende Raum durch seröse Flïssigkeit ausgefüllt wird und unter Umständen der mit dem verdichteten Glaskörper fester zusammenhängende vordere Theil der Netzhaut nach einwärts gezogen ,Werden kann. Diese Veränderung des Glaskörpers, welche mit den senilen Gewebsverdichtungen die grösste Aehnlichkeit hat, während in manchen Fällen auch Proliferationsvorgänge dabei eine grössere Rolle zu spielen scheinen, verdankt ihre Entstehung einer chronischen Entzündung der Aderhaut, deren Nachweis regelmässig erbracht werden konnte.

Hatten diese' sorgfältigen Untersuchungen Nordens on's nun auch unsere Anschauungen über die Entstehung und das Wesen der Netzhautablösung wesentlich geklärt, so hatten sich doch nicht, wie Leber dies gehofft hatte, therapeutische Fortschritte hieran angeknüpft; noch immer stehen wir auf dem gleichen Punkte in dieser Hinsicht, wie vor "unseren pathogenetischen Förderungen dieser Augenaffection, und heute, wie vor Jahren, gilt Leber's Ausspruch: „Die Netzhautablösung ist immer ein ernstes, in der Mehrzahl der Fälle unheilbares Leiden. Fast niemals wird durch Behandlung wirkliche Heilung der Ablösung erzielt."

1) Heft XX, Hamburg und Leipzig, Leopold Voss.
VII. Zur Statistik und Prognose der Herniotomie incarcerirter Hernien im Kindesalter. Von Dr. C. Stern in Dusseldorf.

VIII. Zur Frage der Bedeutung des Auftretens der L oeffler'schen Diphtheriebacillen bei scheinbar gesunden Menschen. Von Dr. P. Aaser in Christiania.

IX. Kritik der Versuche des Herrn Prof. Bruns uber die Wirkung. des Krebsserums. Von Prof. Dr. R. Emmerich und Dr. H. Scholl.

$X$. Therapentische Mittheilungen : Berichtigung zum Inunctor, Salbenreiber von Dr. Aug. Heidenhain (Berlin). Von Dr. Ziemssen in Wiesbaden.

XI. Standesangelegenheiten: Varia.

XII. Kleine Mittheilungen.

Trotz aller bisher bekannten therapeutischen Maassnahmen, deren Aufzählung ich hier unterlasse, haben wir alle immer wieder die Erfahrung machen müssen, dass wir nahezu ohnmächtig dieser Erkrankung gegenüberstehen, wenn auch in seltenen Ausnahmefällen Besserungen, auch wohl Heilungen — selbst spontane beobachtet werden konnten.

Die Heilversuche, die ich auf Grund unserer pathogenetischen Kenntnisse bei der Netzhautablösung unternommen habe, beschäftigen mich seit bereits fünf Jahren, und nachdem ich eine grössere Reihe von Erfahrungen damit gesammelt habe, lege ich sie nunmehr dem ärztlichen Publikum zur Prüfung vor.

Bei meinen ersten therapeutischen Versuchen leitete mich der Gedanke, dass vor allem anderen Verbindungsstränge, welche zwischen einem schrumpfenden Glaskörper und der Netzhaut vermuthet werden müssen, zu durchtrennen seien, dass es gleichzeitig der abgelösten Netzhaut ermöglicht werden müsse, und zwar durch ergiebiges Ablassen der subretinal angesammelten Flüssigkeit, sich an die Aderhaut wieder anzulegen, und dass endlich die Möglichkeit geschaffen werde, dass Netzhaut und Aderhaut mit einander verkleben, resp. an einzelnen Fixationspunkten fest mit einander verwachsen. Diese Bedingungen erfüllt in einem Theile der Krankheitsfälle die von mir sogenannte "Netzhautglaskörperdurchschneidung“. Unter Cocaïnanästhesie des vorher atropinisirten Auges wird mit einem zweischneidigen Linearmesser, mit Verschiebung der Conjunctiva an der Einstichstelle, eine Durchschneidung Augenhäute: Sclera, Choroidea, Retina zunächst an der Stelle der Ablösung vorgenommen; von hier wird das Messer in schräger Richtung quer durch den Glaskörper vorgeschoben, bis es auf der anderen Seite an die Bulbuswand anstösst, und dann mit ganz vorsichtig im Glaskörper nach beiden Seiten hin leicht schneidender Bewegung wieder durch die Eingangsöffnung zurückgezogen. Nach Beendigung des Schnittes wird ein leicht anschliessender antiseptischer Verband auf das operirte Auge gelegt.

Durch dieses Verfahren wird in der That die subretinale Flüssigkeit entleert, die Netzhaut an verschiedenen Stellen eingeschnitten, etwaige Glaskörperstränge, die die Netzhaut halten, werden durchtrennt, auch die präretinale Flüssigkeit wird abgelassen und es der Netzhaut auf diese Weise ermöglicht, sich an die Aderhaut wieder anzulegen; hier wird sie zunächst durch die an den Netzhautdiscissionsstellen auftretende Blutung mit der Aderhaut verklebt gehalten und weiterhin, unter sonst günstigen Umständen, durch Verwachsung dauernd fixirt. Die Patienten bleiben hinterher 8-14 Tage zu Bett, mit leichtem, Verband, bis die Augenspiegeluntersuchung lehrt, dass eine Ablösung der Netzhaut nicht mehr besteht das Auge wird noch einige Wochen nach erfolgter 
Heilung unter Atropinwirkung belassen. Selbst wenn am Tage nach der Operation die Netzhaut noch nicht glatt angelegt ist, so geschieht dies doch in den nächsten Tagen zuweilen spontan; wonn nicht, wird die Operation ein- oder mehre male wiederholt, bis der gew ünschte Effect erreicht ist. Ich habe sechs-, acht-, zehnmal in kurzen Zwischenräumen den Eingriff vorgenommen, wodurch schliesslich 'das gewünschte Resultat, die Heilung, erzielt wurde; gerade durch die Wiederholung ist in einzelnen Fällen der ersehnte Effect überhaupt nur zu ermöglichen. - In einigen wenigen Fällen habe ich eine Verwachsung der Netzhaut und Aderhaut dadurch herbeizufïlıren gesucht, dass ich mit einem spitzen Paquelinbremner Sclera, Choroidea und Retina an der Stelle der Ablösung perforirte; ich bin von dieser Methode wieder abgekommen, weil sie weder allein, noch in Verbindung mit der "Netzhautglaskörperdurchschneidung:" Befriedigendes leistete, sondern durch spätere Narbenretraction wahrscheinlich eher schädlich wirkte. Ich kehrte deshalb zu der einfachen, eventuell öfter. zu wiederholenden Netzhautglaskörperdurchschneidung zurück.

Ich sehe von der Anführung einzelner Krankengeschichten hier $a b$, ich erwähne nur referirend, dass elf Patienten in der geschilderten Weise behandelt wurden, von denen bei vieren die Paquelindurchbohrung gemacht wurde; von letzteren vier gaben zwei Theilerfolge, zwei (darunter eine Totalablösung, an sich hoffnungslos) keinen Erfolg. Bei den sieben nur mit Netzhautglaskörperdurchschneidung behandelten Kranken aber wurden überraschend günstige Resultate erreicht. Es wurde totale Wiederanlegung der Netzhant mit zum Theil sehr gutem Sehvermögen wiedererlangt und die Heilungsdauer noch nach Verlauf von vier Jahren, zwei Jahren, zwei Jahren, zwei Jalren, einem Jahre, fïnf Monaten constatirt; nur in einem Falle wurde eine ninimale Besserung, keine Heilung erlangt, da Patientin sich nur einmal der Operation unterwerfen wollte und weitere Eingriffe ablehnte.

Wie weit dies einfache Verfahren sich num weiterhin nützlich erweisen, d. h. in welchen Fällen es hinreicheu wird, bei genücender Wiederholung eine dauernde Wiederanlegung der abgelösten Netzhaut herbeizufïhren, das muss die weitere Erfahrung und Nachprüfung lehren; es ist selbstverständlich, dass die Methode kein Allheilmittel ist, und ich bin weit entfernt davon, sie als solches anzupreisen; bei anscheinend geeigneten Fällen erreichte ich mit der Netzhautglaskörperdurchschneidung zwar anfänglich günstigen Erfolg, indess keine dauernde Besserung. Die trostlose Empfindung, den thatenlosen Zuschauer bilden zu inüssen bei dem Untergange von Augen, die eine bessere Prognose versprochen hatten, brachte mich zu dem Versuche der Rettung wenigstens eines Theiles solcher Augen auf einem anderen Wege. Die spezielle Veranlassung zu dem Ersinnen dieses Heilverfahrens gab mir ein jugendlicher Patient von 28 Jahren, der auf beiden Augen an Netzhautablösung erkrankte und bei welchem nach Erschöpfung sämmtlicher bekannten Heilmethoden, auch der Netzhautglaskörperdurchschneidung, sich zunächst am linken Auge plötzlich eine Totalablösung der Netzhaut, resp. eine Abreissung derselben, mit Ansammlung blutigseröser Flüssigkeit retroretinal und präretinal einstellte; das Auge war weich, das Sehvermögen bis auf mangelhaften Lichtschein nach oben erloschen.

Ich fasste nun den Entschluss: 1) die blutigseröse Flïssigkeit welche jetzt jedenfalls frei in Bulbus circulirte, abzulassen, 2) den präretinalen Raum mit einer Flüssigkeit anzufüllen, welche die der Aderhaut durch den Strom der abfliessenden Masse möglichst cenäherte Netzhaut hier angedrückt hielt, wenn möglich, auf längere Zeit, 3) die Erregung schwach entzündlicher Vorgänge durch die gleiche einzubringende Flüssigkeit anzubahnen und so eine Verklebung, resp. späterhin Verwachsung von Netzhaut und Aderhaut zu ermöglichen.

Eine solche Flüssigkeit besitzen wir in dein frischen, thierischen Glaskörper, speziell des Kaninchens, Der unter aseptischen Cautelen dem Auge des. lebenden Kaninchens entnommene Glaskörper, von Haus aus selbst aseptisch, erfüllt die vordem postulirten Bedingungen aufs beste. Mit sterilisirter $3 / 4 \%$ iger Chlornatriumlösung lässt er sich bequen in eine Consistenz bringen, die ihn zur Injection in das menschliche Auge geeignet macht; die Verdünnung mit letzterer Lösung lässt uns gleichzeitig den Grad der entzündlichen Reaction abmessen, die er hervorruft. Ich füllte also nach Ablassen der sub- und pröretinalen Flüssigkeit aus dem linken Bulbus des oben erwälnten Kranken mit Hülfe einer Pravaz'schen Spritze in den präretinalen Raum ein wenig frischen Kaninchenglaskörper und hatte die Freude, mein Verfahren von bestem Erfolge gekrönt zu sehen; die Operation wurde am 4. Februar 1894 ausgeführt; am 28. April betrug das Sehvermögen bereits 17/200; am 7. Juli 1894 finde ich notirt $S=17 / 70$. No. 3 Jäger; Se frei. So ist das Sehvermögen dieses Auges geblieben, während sich die Netzhaut tadellos angelegt hatte; auch heute, nach Verlauf von mehr als einem Jahre nach der Operation, ist der Zustand dieses Auges ein unverändert vorzüglicher, wie er oben vom 7. Juli 1894 angegeben ist. Das Auge war nach bestem Wissen als verloren anzusehen und wurde durch die Transplantation von Kaninchenglaskörper wieder in einen Zustand zurüekgeführt, der es einem normalen Auge so nahe als denkbar brachte. Ich gehe auf den Modus der Ausführung dieser Operation, welche immerhin eine technisch recht schwierige ist, hier nicht ein; ich muss für diejenigen Collegen, welche hierfür ein spezielles Interesse haben, auf die ausführlichen Auseinandersetzungen in meiner Arbeit in den Beiträgen zur Augenheilkunde verweisen. Ich hebe nur referirend, auch ohne auf die Einzelcasuistik der von mir derartig behandelten Fälle einzugehen, noch hervor, dass von mir im ganzen sechs Patienten und sieben Augen in der angegebenen Weise behandelt worden sind, und zwar nur Augen, die bereits bis auf Lichtschein oder ein nicht inehr als "Sehen" in Betracht kommendes Sellvermögen erblindet waren. "Bei vier in solchem Zustande noch meiner Therapie unterworfenen Augen ist ein Erfolg erzielt worden, wie er mir vorher als unmöglich hätte scheinen müssen, wenn ich mich an die Kenntniss dessen gehalten hätte, was wir bis dahin über die Möglichkeit der Heilung totaler Netzhautablösung wussten.

Bei Fall 2 war der Sachlage nach unter keiner Bedingung: mehr zu erwarten, als erreicht wurde; bei jahrelangem Bestand der Solutio, Cataracta incipiens, excentrischem Fingerzählen in 4 Fuss, wurde ein kleines, selrr bescheidenes centrales Sehen, bei Verdoppelung des excentrischen und Heilung der Ablösung wiedergewonnen; bei Fall 3, einer totalen Solutio nach Traunra, leistete die Methode so gut wie nichts, insofern das Sehvermögen nur auf excentrisches Fingerzählen in 2-3 Fuss gebracht werden kounte bei fast total weiterbestehender Netzhautablösung, wälrend es vor jedem Hingriff auf Handbewegungen reducirt gewesen war. In den übrigen Fällen aber war der Erfolg ein überaus giüstiger. nnd die Beobachtungsdauer erstreckt sich hier bei Fall 1, bei dem das beste Resultat erlangt wurde, bereits auf über ein Jalrr; bei Fall 2 auf drei Vierteljahre, bei Fall 5 auf über ein halbes Jahr, bei Fall 6 auf über fünf Monate nach erreichter Anlegung der Netzhant.

So wenig, wie auf den Modus dieser von mir als Kaninchenglaskörpertransplantation bezeichneten Operation, kann ich hier auf den Verlauf nach Ausführung derselben näher eingehen; es sei nur kurz erwähnt, dass dem Eingriff ein reactiv entzïndliches Stadium folgt, das sich leicht in Schranken halten lässt und das unbedingt eintreten muss, wenn anders es zu einer dauernden Verwachsung von Netzhaut und Aderhaut kommen soll. Bei etwaigem Misserfolg lässt sich das Verfahren in kürzerer oder längerer Zeit, je nach dem Zustande des betreffenden Auges, wiederholen, um dann bei zweiter oder dritter Anwendung schliesslich doch zu dem gewünschten Heileffect zu fülren.

Unzweifelhaft wird es vorkommen, dass auch dieses Heilverfahren bei Netzhautablösung in Stiche lassen wird, das liegt in der Natur der Erkrankung; andererseits wird es ebenso unzweifelhaft dann noch einen Theil von Augen retten, wenn kein uns bekanntes Heilmittel instande war, dieselben vor der sonst sicheren Erblindung zu bewahren. Wird aber durch diese Behandlungsmethode, auch den ungünstigen Fall eines späteren Recidivs angenommen, die Erblindung auf einige Jahre hinausgeschoben, so wäre dies ein für derartige Unglïckliche sicher nicht zu unterschätzender Gewinn.

Unter allen Uniständen darf man natürlich auch von dieser Operation nicht inehr verlangen, als sie günstigsten Falles zu leisten imstande ist; sie wird ebenso wenig eine degenerirte Netzhaut wieder zu einer normal functionirenden machen, wic irgend eine andere Behandlungsmethode: sie wird daher unter Uniständen vielleicht bezüglich des Sehvermögens, das sie wiedergeben kann, vorher gehegte Erwartungen tänschen, wie sie solche andernfalls bei weitem übertreffen kann. Da sie indess zumeist an sonst der sicheren Erblindung überlieferten Augen ihre Anwendung finden wird, so kann durch sie nichts mehr verloren, wohl aber relativ unendlich viel gewonnen werden. 